\title{
Tramadol efficacy in patients with postoperative pain in relation to CYP2D6 and MDR1 polymorphisms
}

\author{
Slanar $\mathrm{O}^{1}$, Dupal $\mathrm{P}^{2}$, Matouskova $\mathrm{O}^{1}$, Vondrackova $\mathrm{H}^{1}$, Pafko $\mathrm{P}^{3}$, Perlik $\mathrm{F}^{1}$ \\ Institute of Pharmacology, First Faculty of Medicine and General Teaching Hospital, Charles University \\ in Prague, Czech Republic. oslan@If1.cuni.cz
}

\begin{abstract}
Objectives: The aim of our study was to evaluate impact of CYP2D6 and MDR1 polymorphisms on the analgesic efficacy of tramadol in patients after a knee arthroscopy.

Background: Pharmacokinetics of tramadol and its metabolites is stereoselective and displays high interindividual variability correlating with polymorphic CYP2D6 in the population. Available data provide controversial results regarding the analgesic efficacy of tramadol in subjects with different CYP2D6 genotypes.

Methods: Pain intensity was assessed using visual analogue scale at 2 and 24 hours after the knee arthroscopy in 156 patients. Polymorphisms CYP2D $6{ }^{*} 3,{ }^{*} 4,{ }^{*} 5,{ }^{*} 6$, and gene duplication and C3435T in MDR1 gene were analyzed by PCR - RFLP.

Results: Mean $\mathrm{VAS}_{2 \mathrm{~h}}$ value in the whole study group was $44.0 \pm 16.5 \mathrm{~mm}$. Mean pain difference, was lowest in the UM group and highest in the PM group. The pain difference varied significantly among the CYP2D6 subgroups ( $F=4.29 ; p=0.006)$ with significant differences between homEM vs hetEM, homEM vs PM, and UM vs PM subgroups. There were no significant differences among MDR1 subgroups with regards of pain difference. Mean tramadol consumption was $2.47 \pm 1.17 \mathrm{mg} / \mathrm{kg}$ during the $24 \mathrm{~h}$ period. There were no significant differences in the drug consumption, reporting of adverse reactions, need for rescue analgesic medication or verbal description of pain among the CYP2D6 or MDR1 genotype subgroups.

Conclusion: CYP2D6 plays a significant role in tramadol analgesic efficacy. The non-opioid analgesia in PMs was associated with better subjective pain relief in patients after a knee arthroscopy (Tab. 3, Ref. 18). Full Text in PDF www.elis.sk.

Key words: analgesia, tramadol, acute pain, polymorphism, arthroscopy.
\end{abstract}

\begin{abstract}
Abbreviations: MDR1 - Multidrug resistence 1, VAS - Visual analogue scale, DNA - Deoxyribonucleic acid, PCR - RFLP - Polymerase chain reaction - restriction fragment length polymorphism, homEM - homozygous extensive metabolizer, hetEM - heterozygous extensive metabolizer, PM - poor metabolizer, UM - ultrarapid metabolizer, CYP2D6 - cytochrome P450 2D6.

Tramadol is a frequently used analgesic drug used for the treatment of acute postoperative pain. In the treatment of mild to moderate pain, tramadol is as effective as morphine, while for the treatment of severe or chronic pain the efficacy of tramadol is lower in comparison with morphine.
\end{abstract}

${ }^{1}$ Institute of Pharmacology, First Faculty of Medicine and General Teaching Hospital, Charles University in Prague, Czech Republic, ${ }^{2}$ Department of Orthopaedics, First Faculty of Medicine and University Hospital Motol, Charles University in Prague, Czech Republic, and ${ }^{3}$ Third Department of Surgery, First Faculty of Medicine and University Hospital Motol, Charles University in Prague, Czech Republic

Address for correspondence: $\mathrm{O}$. Slanar, $\mathrm{MD}, \mathrm{PhD}$, Institute of Pharmacology, 1st Faculty of Medicine and General Teaching Hospital, Albertov 4, CZ-128 00 Prague 2, Czech Republic.

Phone: +420.2 .24968104$

Acknowledgement: This study has been supported by a GAČR grant No. 305/08/P069.
The metabolic fate of tramadol is unusually complex having at least 11 unconjugated metabolites and 12 conjugated compounds (1). There are three major metabolic pathways via three distinct cytochrome P450 enzymes CYP2D6, CYP3A, and CY$\mathrm{P} 2 \mathrm{~B} 6$ forming $\mathrm{O}-$ and $\mathrm{N}-$ demethylated metabolites. Major active metabolite O-demethyltramadol is, however, formed in the liver predominantly via CYP2D6 enzyme $(1,2)$. Pharmacokinetics of tramadol and its metabolites is indeed stereoselective and displays high interindividual variability correlating with polymorphic activity of CYP2D6 in the population (3-7). Further, our previous data suggest, that the pharmacokinetics of tramadol could be also affected by MDR1 polymorphism C3435T (8).

The drug belongs among weak $\mu$-opioid agonists with affinity to the $\mu$-receptor being only 1:6000 that of morphine. Tramadol is supplied as the racemate for therapy, but studies with cloned human $\mu$-opioid receptor have revealed that $(+)$-M1 has approximately 200-times the affinity of the parent $( \pm)$-tramadol (9). Production of the (+)-M1 metabolite from $(+)$-tramadol is therefore considered essential for opioid activity. In addition, there is good evidence that non-opioid mechanisms are involved in the analgesic properties of tramadol, in particular inhibition of neuronal reuptake of both noradrenaline and 5-HT. In this case, (-)-tramadol and $(+)$-tramadol are potent inhibitors of noradrenaline and 5-HT reuptake, respectively (10). 
Both opioid and non-opioid analgesic components contribute synergistically to tramadol-induced antinociception (10).

Given the polymorphic CYP2D6 dependent pharmacokinetics the drug mechanism of action, variable clinical efficacy influenced by CYP2D6 activity could be foreseen. However, available clinical data provide controversial results regarding the analgesic efficacy of tramadol in subjects with different CYP2D6 phenotype/genotype. Some clinical studies revealed decreased analgesic efficacy in CYP2D6 poor metabolizers (11) in line with experimental model of pain (12), while inhibition of CYP2D6 by escitalopram did not impair the analgesic effect of tramadol in another study although there was a shift in drug pharmacokinetics confirming decreased CYP2D6 (13). This is in line with different observations in pain model in healthy volunteers, in which PM subjects reported analgesia during electrical stimulation test (14).

The aim of our study was to evaluate possible impact of CYP2D6 and MDR1 polymorphisms on the analgesic efficacy of tramadol in realistic clinical settings in patients after a knee arthroscopy.

\section{Materials and methods}

Totally 225 patients elected to undergo knee arthroscopy were included into this study when signing the inform consent. Exclusion criteria were (i) severe liver and kidney disease, (ii) depression, (iii) anxiety, (iv) chronic use of analgesics (v) administration of non-steroidal anti-inflammatory analgesics and/or opiates one week before the surgery, (vi) use of co-medications potentially interacting with tramadol pharmacokinetics or pharmacodynamics.

The study was approved by the local Ethics Committee and it was conducted in accordance with the Declaration of Helsinki.

All patients underwent surgery under spinal anaesthesia with bupivacaine (Marcaine Spinal 0.5 \%, AstraZeneca UK, Ltd.). Tramadol (Tralgit inj., Zentiva a.s., Slovakia) was the only analgesics used after returning the patient from the surgery room. The drug was given on demand intramuscularly (Tralgit inj., Zentiva a.s., Slovakia) at a dosage of $100 \mathrm{mg}$ for 1 application or orally (Tralgit $50 \mathrm{mg}$, Zentiva a.s., Slovakia) $50 \mathrm{mg}$ in immediate release formulation. Pain intensity was assessed using visual analogue scale (VAS 0-100 mm) at 2 and 24 hours after the surgery. Patients with initial pain less than $20 \mathrm{~mm}$ were excluded from evaluation in this study. In addition, patient's verbal description of pain using standardised short version of McGill Pain Questionnaire was evaluated. Analgesic consumption, need for escape analgesic therapy, possible adverse effects were also monitored.

Samples of peripheral venous blood for DNA isolation were collected in tubes containing $\mathrm{K}_{2}$ EDTA and immediately frozen and stored at $-20{ }^{\circ} \mathrm{C}$ until further processing. DNA was subsequently isolated using QIAamp DNA Blood Mini Kit (Quiagen Ltd.). Detection of the most prevalent functional polymorphisms in the Czech population in CYP2D6 and polymorphism C3435T in MDR1 gene was performed by PCR - RFLP analysis, as published previously $(15,16)$. Phenotype of CYP2D6 was predicted based on the number of variant alleles within the gene. Subjects carrying two inactive alleles were classified as poor metabolizers
(PM), patients with one active and one variant allele were classified as heterozygous extensive metabolizers (hetEM) and subjects with no variant and two active alleles were assigned homozygous extensive metabolizer (homEM) phenotype. Subjects with more than 2 active alleles formed group of ultrarapid metabolizers (UM). Technicians responsible for genotyping were blinded regarding patient's clinical outcome. One-way ANOVA, Kruskal-Wallis and Multiple range tests were used for statistical evaluation of differences between the genotype groups. $\chi^{2}$-test was used for comparison of number of responders and non-responders. Differences were considered statistically significant for $\mathrm{p}<0.05$.

\section{Results}

Sixty-nine patients have been excluded from evaluation due to non-compliance, loss of follow-up or initial VAS value less than $20 \mathrm{~mm}$. Among the evaluable 156 patients, homEM, hetEM, PM, and UM phenotypes were predicted in $86,55,9$, and 6 patients, respectively. There were 22 wild-type homozygous subjects, 86 patients were heterozygotes, and 48 patients carried variant allele only in the C3435T polymorphism. The most frequent CYPD6 variant allele was CYP2D6*4 with allelic frequency of approximately $20 \%$, while the other functionally variant alelles occurred with frequency lower that $4 \%$. Variant allele $3435 \mathrm{~T}$ in the MDR 1 gene was seen in frequency of approximately $58 \%$. The frequencies of the variant alleles in the study group are shown in the Table 1. The distribution of variant alleles corresponded to the HardyWeinberg equilibrium.

Mean $( \pm \mathrm{SD})$ age and BMI of the group was $39.6 \pm 14.2$ years and $25.8 \pm 4.3 \mathrm{~kg} / \mathrm{m}^{2}$, respectively. There were no statistically significant differences between CYP2D6 and MDR1 genotype subgroups in basic demographic parameters, but we noted higher mean age $49.2 \pm 11.8$ years in the UM group as compared to other CYP2D6 phenotype groups. Basic demographic description of the group is shown in the Table 2 .

Tab. 1. Frequency of variant alleles in the study group.

\begin{tabular}{lccc}
\hline Gene & Allele & Allelic frequency $(\%)$ & $95 \%$ CI \\
\hline CYP2D6 & $* 3$ & 1.0 & $0.33-2.79$ \\
& $* 4$ & 20.2 & $16.11-25.00$ \\
& $* 5$ & 3.5 & $1.98-6.20$ \\
& $* 6$ & 0.0 & $0.00-1.20$ \\
& duplication & 2.9 & $1.52-5.39$ \\
\hline MDR1 & $3435 \mathrm{~T}$ & 58.3 & $52.79-63.67$ \\
\hline
\end{tabular}

Tab. 2. Demographic data of patients in CYP2D6 and MDR1 genotype subgroups (mean \pm S.D.; number of subjects).

\begin{tabular}{llccc}
\hline Gene & $\begin{array}{l}\text { Predicted } \\
\text { phenotype/ } \\
\text { genotype }\end{array}$ & $\begin{array}{c}\text { Age } \\
\text { (years) }\end{array}$ & $\begin{array}{c}\text { BMI } \\
\left(\mathrm{kg} / \mathrm{m}^{2}\right)\end{array}$ & $\begin{array}{c}\text { Male to } \\
\text { female ratio }\end{array}$ \\
\hline CYP2D6 & homEM $(\mathrm{n}=86)$ & $39.1 \pm 14.5$ & $25.5 \pm 4.4$ & $42: 44$ \\
& hetEM $(\mathrm{n}=55)$ & $39.7 \pm 13.9$ & $25.4 \pm 3.7$ & $37: 18$ \\
& PM $(\mathrm{n}=9)$ & $36.9 \pm 15.1$ & $26.9 \pm 5.1$ & $4: 5$ \\
& UM $(\mathrm{n}=6)$ & $49.2 \pm 11.8$ & $29.3 \pm 6.1$ & $3: 3$ \\
\hline MDR1 & 3435CC $(\mathrm{n}=22)$ & $42.4 \pm 13.7$ & $25.6 \pm 5.1$ & $10: 12$ \\
& 3435CT $(\mathrm{n}=86)$ & $40.0 \pm 14.7$ & $25.7 \pm 4.4$ & $46: 40$ \\
& 3435TT $(\mathrm{n}=48)$ & $37.5 \pm 13.5$ & $25.6 \pm 3.9$ & $30: 18$ \\
\hline
\end{tabular}


Tab. 3. Comparison of pain characteristics and tramadol consumption in CYP2D6 and MDR1 genotype subgroup over 24 hours postoperative period. Data are presented as mean \pm S.D.

\begin{tabular}{llccc}
\hline Gene & $\begin{array}{l}\text { Predicted } \\
\text { phenotype/ } \\
\text { genotype }\end{array}$ & $\begin{array}{c}\text { Initial pain } \\
\text { intensity } \\
\mathrm{VAS}_{2 \mathrm{~h}}(\mathrm{~mm})\end{array}$ & $\begin{array}{c}\text { Pain difference } \\
\mathrm{VAS}_{2-24 h} \\
(\mathrm{~mm})\end{array}$ & $\begin{array}{c}\text { Total tramadol } \\
\text { consumption } \\
(\mathrm{mg} / \mathrm{kg} / 24 \mathrm{~h})\end{array}$ \\
\hline CYP2D66 & homEM $(\mathrm{n}=86)$ & $41.3 \pm 15.9$ & $18.8 \pm 13.4$ & $2.39 \pm 1.4$ \\
& hetEM $(\mathrm{n}=55)$ & $44.7 \pm 17.1$ & $24.8 \pm 15.0$ & $2.54 \pm 1.2$ \\
& PM $(\mathrm{n}=9)$ & $52.1 \pm 18.8$ & $33.2 \pm 15.7$ & $2.48 \pm 1.3$ \\
& UM $(\mathrm{n}=6)$ & $51.0 \pm 15.7$ & $18.0 \pm 12.0$ & $2.56 \pm 0.8$ \\
MDR1 & 3435CC $(\mathrm{n}=22)$ & $40.0 \pm 11.8$ & $19.3 \pm 12.1$ & $2.62 \pm 1.1$ \\
& 3435CT $(\mathrm{n}=86)$ & $43.2 \pm 17.9$ & $21.3 \pm 14.6$ & $2.42 \pm 1.1$ \\
& 3435TT $(\mathrm{n}=48)$ & $45.5 \pm 16.1$ & $23.4 \pm 15.4$ & $2.44 \pm 1.3$ \\
\hline
\end{tabular}

Table 3 shows initial pain intensity, pain difference and tramadol consumption in the genotype subgroups. Mean initial VAS2 $h$ value in the whole study group was $44.0 \pm 16.5 \mathrm{~mm}$. Although the PM and UM subjects reported slightly higher mean pain intensity values of $52.1 \pm 18.8$ and $51.0 \pm 15.7 \mathrm{~mm}$, respectively, the differences among genotype subgroups have not been statistically significant. MDR1 genotype subgroups reported mean initial VAS2h values insignificantly deviating from the mean value of the whole study group by $4.0 \mathrm{~mm}$ or less. Mean pain difference, defined as VAS2h - VAS24h, was lowest in the UM group and highest in the PM group. The pain difference varied significantly among the CYP2D6 subgroups (ANOVA F=4.29; $\mathrm{p}=0.006$ ). Multiple range test revealed significant differences between homEM vs hetEM, homEM vs PM and UM vs PM subgroups. There were no significant differences among MDR1 subgroups with regards of pain difference. Mean tramadol consumption in whole study group was $2.47 \pm 1.17 \mathrm{mg} / \mathrm{kg}$ during the $24 \mathrm{~h}$ post-surgery period. There were no significant differences in the drug consumption among the CYP2D6 or MDR1 genotype subgroups.

We noted no substantial differences among genotype subgroups in reporting of adverse reactions, need for rescue analgesic medication or verbal description of pain. The treatment was overall well tolerated, 6 patients complained from nausea and vomiting as the most probable adverse drug reactions while only 7 patients required additional rescue analgesic therapy.

\section{Discussion}

This study has been conducted in a group of patients after a knee arthroscopy serving as a clinical model for mild to moderate pain, which is an appropriate indication for tramadol use. Our study population displayed similar distribution of CYP2D6 predicted phenotypes and MDR1 genotypes to those in the Czech healthy population $(15,16)$.

In case of arthroscopy, mean reported pain reaches the highest intensity shortly after the surgery (at 2 hours) with a subsequent plateau till approximately 8 hours post-surgery and declines afterwards (17). Therefore we designed this observational study to detect the peak initial VAS value at 2 hours with further VAS measurement at the end of overnight hospitalization. Mean initial pain intensity $44 \mathrm{~mm}$ in the evaluable group fits well within the expected VAS value for moderate pain. Tramadol consumption was rather low in our study reaching approximately $2.5 \mathrm{mg} / \mathrm{kg}$ per 24 hours, which probably reflects decreasing pain intensity in the period 8-24 hours after surgery and generally low VAS score at 24 hours post-surgery. There were no substantial differences among genotype subgroups with regards of drug consumption, which could be probably detectable only if there were more pronounced genotype-specific variability in pain intensity or pain differences.

Although there were approximately $20 \%$ higher mean pain difference values in the 3435TT group in comparison with the wild type subjects, the between group variation did not reach statistical significance. The 3435TT genotype has been previously reported to lead to the lowest P-glycoprotein expression (18). Potentially higher drug disposition to the brain in the homozygous carriers of 3435T allele could represent the background for our observation. Statistically significant genotype-related differences were observed only for CYP2D6. The pain difference between 24 and 2 hours post-surgery was lowest in UM group, followed by homEM towards hetEM and PM subjects. The lower the predicted CYP2D6 activity was, the more pronounced decrease in pain intensity was observed. One could speculate that the pain intensity in our study group was sufficiently low for non-opioid mechanisms of tramadol-induced analgesia, which would not be affected by CYP2D6 deficit since being mediated primarily by the parent compound. There were no significant differences in initial VAS scores among the genotype subgroups and slightly higher reported pain intensity in PM and UM groups do not seem affect the interpretation of pain difference data.

The results of our study suggest that CYP2D6 plays a role in tramadol analgesic efficacy. The clinical relevance of being poor, extensive, or ultrarapid metabolizer for CYP2D6, however, seem to differ in various pain intensity situations, where for less severe pain stimuli the non-opioid analgesia by parent compound in PMs may lead to better subjective pain relief, while in case of more severe painful conditions lack of opioid agonistic activity in PMs could lead to decreased analgesic efficacy.

\section{Conclusion}

CYP2D6 plays a significant role in tramadol analgesic efficacy. The non-opioid analgesia by parent compound in PMs was associated with better subjective pain relief in our study in patients after a knee arthroscopy.

\section{References}

1. Wu WN, McKown LA, Liao S. Metabolism of the analgesic drug ULTRAM (tramadol hydrochloride) in humans: API-MS and MS/MS characterization of metabolites. Xenobiotica 2002; 32 (5): 411-425.

2. Paar WD, Frankus P, Dengler HJ. The metabolism of tramadol by human liver microsomes. Clin Investig 1992; 70 (8): 708-710.

3. Slanar O, Nobilis M, Kvetina J, Idle JR, Perlik F. CYP2D6 polymorphism, tramadol pharmacokinetics and pupillary response. Eur J Clin Pharmacol 2006; 62 (1): 75-76; author reply 77-78.

4. Chytil L, Sticha M, Matouskova O, Perlik F, Slanar O. Enatiomeric determination of tramadol and O-desmethyltramadol in human urine by 
gas chromatography-mass spectrometry. J Chromatogr B Analyt Technol Biomed Life Sci 2009; 877 (20-21): 1937-1942.

5. Chytil L, Matouskova O, Cerna O, Pokorna P, Vobruba V, Perlik F, Slanar O. Enantiomeric determination of tramadol and O-desmethyltramadol in human plasma by fast liquid chromatographic technique coupled with mass spectrometric detection. J Chromatogr B Anlyt Technol Biomed Life Sci 2010; 878 (3-4): 481-486.

6. Gan SH, Ismail R, Wan Adnan WA, Zulmi W. Impact of CYP2D6 genetic polymorphism on tramadol pharmacokinetics and pharmacodynamics. Mol Diagn Ther 2007; 11 (3): 171-181.

7. Borlak J, Hermann R, Erb K, Thum T. A rapid and simple CYP2D6 genotyping assay - case study with the analgetic tramadol. Metabolism 2003; 52 (11): 1439-1443.

8. Slanar O, Nobilis M, Kvetina J, Matouskova O, Idle JR, Perlik F. Pharmacokinetics of tramadol is affected by MDR1 polymorphism C3435T. Eur J Clin Pharmacol 2007; 63 (4): 419-421.

9. Gillen C, Haurand M, Kobelt DJ, Wnendt S. Affinity, potency and efficacy of tramadol and its metabolites at the cloned human mu-opioid receptor. Naunyn Schmiedebergs Arch Pharmacol 2000; 362 (2): 116-121.

10. Raffa RB, Friderichs E, Reimann W, Shank RP, Codd EE, Vaught JL, Jacoby HI, Selve N. Complementary and synergistic antinociceptive interaction between the enantiomers of tramadol. J Pharmacol Exp Ther 1993; 267 (1): 331-340.

11. Stamer UM, Lehnen K, Hothker F, Bayerer B, Wolf S, Hoeft A, Stuber F. Impact of CYP2D6 genotype on postoperative tramadol analgesia. Pain 2003; 105 (1-2): 231-238.
12. Poulsen L, Arendt-Nielsen L, Brosen K, Sindrup SH. The hypoalgesic effect of tramadol in relation to CYP2D6. Clin Pharmacol Ther 1996; 60 (6): 636-644.

13. Noehr-Jensen L, Zwisler ST, Larsen F, Sindrup SH, Damkier P, Brosen K. Escitalopram is a weak inhibitor of the CYP2D6-catalyzed O-demethylation of (+)-tramadol but does not reduce the hypoalgesic effect in experimental pain. Clin Pharmacol Ther 2009; 86 (6): 626-633.

14. Enggaard TP, Poulsen L, Arendt-Nielsen L, Brosen K, Ossig J, Sindrup SH. The analgesic effect of tramadol after intravenous injection in healthy volunteers in relation to CYP2D6. Anesth Analg 2006; 102 (1): $146-150$.

15. Buzkova H, Pechandova K, Slanar O, Perlik F. Frequency of single nucleotide polymorphisms of CYP2D6 in the Czech population. Cell Biochem Funct 2008; 26 (1): 76-81.

16. Pechandova K, Buzkova H, Slanar O, Perlik F. Polymorphisms of the MDR1 gene in the Czech population. Folia Biol (Praha) 2006; 52 (6): 184-189.

17. Drosos GI, Stavropoulos NI, Katsis A, Kesidis K, Kazakos K, Verettas DA. Post-operative pain after knee arthroscopy and related factors. Open Orthop J 2008; 2: 110-114.

18. Song P, Lamba JK, Zhang L, Schuetz E, Shukla N, Meibohm B, Yates CR. G2677T and C3435T genotype and haplotype are associated with hepatic ABCB1 (MDR1) expression. J Clin Pharmacol 2006; 46 (3): 373-379.

Received January 18, 2011. Accepted January 20, 2012. 